\title{
INFLUÊNCIA DO OCTREOTIDE SUBCUTÂNEO NA CICATRIZAÇÃO DE SUTURA GÁSTRICA EM RATOS
}

\section{Effect of octreotide administered subcutaneously on the healing of gastric sutures in rats}

\author{
Paula Suzin TRUBIAN, Alessandra Borges BRANCO Antonio Carlos Ligocki CAMPOS, Marcelo FERREIRA, \\ Patrícia Suzin TRUBIAN, Jorge Eduardo Fouto MATIAS
}

ABCDDV/553

Trubian PS, Branco AB, Campos ACL, Ferreira M, Trubian PS, Matias JEF. Influência do octreotide subcutâneo na cicatrização de sutura gástrica em ratos. ABCD Arq Bras Cir Dig 2007;20(3):172-8.

RESUMO - Racional - Octreotide, análogo sintético da somatostatina, é potente inibidor da secreção gástrica, intestinal e pancreática. Reduz fluxo sangüíneo esplâncnico, acelera esvaziamento gástrico e prolonga tempo de trânsito intestinal, estimulando a absorção de água e eletrólitos. Freqüentemente é utilizado no tratamento das fístulas digestivas pela sua capacidade de reduzir o débito da fístula, porém a redução do fluxo sangüíneo poderia interferir negativamente na cicatrização. Objetivo -Avaliar a influência do octreotide na cicatrização de sutura gástrica em ratos. Métodos - Foram utilizados 39 ratos Wistar machos, divididos em dois grupos, controle e octreotide, subdivididos em 4 e 7 dias, de acordo com o dia da morte do animal. Todos foram submetidos à gastrotomia e fechamento com pontos seromusculares interrompidos. Os animais do grupo octreotide receberam dose única diária do medicamento por via subcutânea. O grupo controle recebeu solução salina a $0,9 \%$. Foram determinados o percentual de colágeno maduro e imaturo, pela técnica de picrosirius-red F3BA. O cálculo do índice de maturação do colágeno e determinação da força de ruptura (FRT) e força máxima de tração (FMT), foi testada em tensiômetro computadorizado. Utilizou-se para análise estatística, os testes $t$ de Student e KruskalWallis com nível de significância de $P<0,05$. Resultados - Na avaliação do colágeno imaturo não houve diferença estatisticamente significativa. Quanto ao maduro, no grupo controle, houve maior percentual no subgrupo do $7^{\circ}$ dia pós-operatório $(21,43 \pm 1,06$ vs.11,26 $\pm 1,44, P=0,0000)$. No grupo octreotide, não houve diferença entre os subgrupos. Na avaliação inter-grupos do colágeno maduro, a dosagem foi maior no $4^{\circ}$ dia do pósoperatório do grupo octreotide $(19,13 \pm 1,38$ vs. $11,26 \pm 1,44, P=0,001)$. O índice de maturação do colágeno maior para o grupo octreotide no $4^{\circ}$ dia do pós-operatório $(1,07 \pm 0,07$ vs. $0,65 \pm 0,05, P=0,0001)$. A FMT e a FRT não apresentaram diferença entre os grupos. Conclusões - A administração de octreotide por via subcutânea aumenta a deposição de colágeno maduro e não altera a resistência tênsil de gastrorrafias em rat

DESCRITORES - Octreotide. Colágeno. Sutura.

\section{INTRODUÇÃO}

Concomitantemente com a introdução de medicamentos potentes, tecnologia avançada de diagnósticos e novos tratamentos conservadores, muitas operações gastrointestinais tornaram-se desnecessárias, em particular procedimentos cirúrgicos ácido-redutores sobre o estômago. Em contrapartida, as operações sobre o estômago cresceram de forma exponencial com o desenvolvimento da cirurgia bariátrica. Como o tratamento do câncer gástrico permanece sendo fundamentalmente cirúrgico, pode-se inferir que o conhecimento do processo de cicatrização gástrica permanece relevante na prática do cirurgião digestivo.

Durante as décadas de 50 e 60, as pesquisas foram direcionadas ao estudo dos fatores de ordem técnica capazes de interferir na cicatrização. Estas pesquisas contribuíram com alguns conceitos, hoje universalmente aceitos, como a importância da inversão ou eversão das paredes na

Trabalho realizado no Departamento de Cirurgia da Universidade Federal do Paraná, Curitiba, PR, Brasil.

Endereço para correspondência: Antonio Carlos Campos, e-mail: accampos@hc.ufpr.br linha de sutura para melhorar a coaptação dos bordos e a necessidade de inclusão da submucosa nos pontos, por ser esta camada rica em colágeno ${ }^{21}$. Recentemente houve crescente interesse nos efeitos dos agentes farmacológicos sobre a cicatrização, uma vez que complicações na cicatrização de anastomoses, como as fístulas, também causam aumento dos custos médicos, hospitalização prolongada e diminuição da qualidade de vida do paciente $^{2}$. Outra condição clínica, também responsável por altas taxas de morbimortalidade e grande volume de hospitalizações de emergência, é o sangramento digestivo alto ${ }^{19}$. Não são infreqüentes complicações pós-operatórias como fístulas gástricas com ou sem sangramento, ou situações em que o paciente está fazendo uso de octreotide e acaba por necessitar de algum procedimento cirúrgico gástrico (sangramento ou ressangramento, neoplasias, carcinomatoses $)^{2,8,14}$.

O octreotide é um octapeptídio sintético da somatostatina, que tem alta potência e longa duração de ação. É efetivo depois da administração subcutânea e o efeito rebote de hipersecreção observado com a somatostatina não se repetiu com esta substância ${ }^{8}$. Os efeitos farmacológicos do octreotide incluem a inibição de numerosos hormônios de secreções exócrinas e da absorção no intestino delgado, as- 
sim como a redução no fluxo sangüíneo portal. $\mathrm{O}$ fato de reduzir de maneira bastante efetiva as secreções digestivas fez do octreotide importante agente farmacológico adjuvante no tratamento das fístulas digestivas. Entretanto, como o seu uso se associa à redução do fluxo sanguíneo portal, e tendo-se em conta a importância da boa vascularização para a cicatrização de suturas gastrointestinais, pode-se inferir que ele poderia teoricamente interferir negativamente no processo de cicatrização de suturas gástricas.

O presente estudo teve por objetivo investigar os efeitos do octreotide na cicatrização de sutura gástrica em ratos avaliados no $4^{\circ}$ e $7^{\circ}$ dia do pós-operatório, mediante a avaliação dos seguintes parâmetros: 1. força máxima de tração e da ruptura das suturas gástricas; 2 . deposição de colágeno e na sua distribuição qualitativa.

\section{MÉTODOS}

Este estudo faz parte da linha de pesquisa em Cicatrização de Órgãos e Tecidos do Programa de Pós-Graduação em Clínica Cirúrgica da Universidade Federal do Paraná (UFPR) e foi realizado de acordo com as normas de pesquisa animal dispostas na legislação nacional e obedeceu aos Princípios Éticos na Experimentação Animal postulados pelo Colégio Brasileiro de Experimentação Animal (COBEA). O experimento foi realizado no Laboratório de Cirurgia Experimental do Programa de Pós-Graduação em Clínica Cirúrgica da UFPR. As condições ambientais foram controladas eletronicamente, sendo mantida temperatura de $22 \pm 1^{\circ} \mathrm{C}$ e ciclo claro-escuro de 12 horas. Os animais foram identificados e mantidos em caixas de plástico com tampa de aço inoxidável, contendo no máximo quatro animais por caixa. Durante o período de aclimação de 5 dias antes do início do experimento, os ratos receberam água potável e ração ad libitum.

Foram utilizados 44 ratos Wistar machos (Rattus norvegigus albinus, Rodentia mammalia), adultos, com peso inicial médio de 255,95 $\pm 3,03 \mathrm{~g}$. Após o período de aclimação, foram pesados e divididos aleatoriamente em dois grupos de acordo com o tipo de substância aplicada durante o experimento: grupo controle $(\mathrm{C})$ e grupo octreotide $(\mathrm{O})$. Estes dois grupos foram divididos em dois subgrupos de acordo com o dia de morte do animal: 4 e 7 dias. Os animais pertencentes ao grupo octreotide receberam injeção única diária de octreotide na dose de $20 \mu \mathrm{g} / \mathrm{kg} / \mathrm{dia}$, por via subcutânea e os do grupo controle receberam injeção de solução salina isotônica $0,9 \%$, isovolumétrica diariamente por via subcutânea. Em ambos os casos, a dose inicial foi aplicada $12 \mathrm{~h}$ antes do procedimento cirúrgico e foi repetida diariamente até a data do óbito.

O medicamento Octreotide (Sandostatin ${ }^{\circledR}$, Novartis, Suíça) foi utilizado na forma injetável e conservado em temperatura inferior a $8^{\circ} \mathrm{C}$. Para a diluição do medicamento, utilizou-se, solução salina isotônica, conforme orientação do fabricante.

Os animais foram submetidos à anestesia inalatória com éter etílico e a manutenção foi realizada com hidrato de cloral $10 \%$ intraperitoneal. Em seguida foram fixados à mesa cirúrgica, degermados com polivinilpirrolidona- iodo (PVP-I) e tricotomizados. Realizou-se laparotomia mediana de cerca de $4 \mathrm{~cm}$ de comprimento para dar acesso à cavidade abdominal, a seguir realizou-se o inventário da cavidade e exposição do estômago do rato. Foi realizada abertura de $30 \mathrm{~mm}$ na curvatura maior do estômago entre o rúmen e o corpo. A gastrorrafia foi realizada com pontos simples, seromusculares, com distância de $2 \mathrm{~mm}$ entre eles, com fio inabsorvível de polipropileno 5-0 (Figura 1). $\mathrm{O}$ fechamento da parede foi realizado com sutura contínua de fio monofilamentar inabsorvível 3-0 em dois planos: aponeurótico e subcuticular.

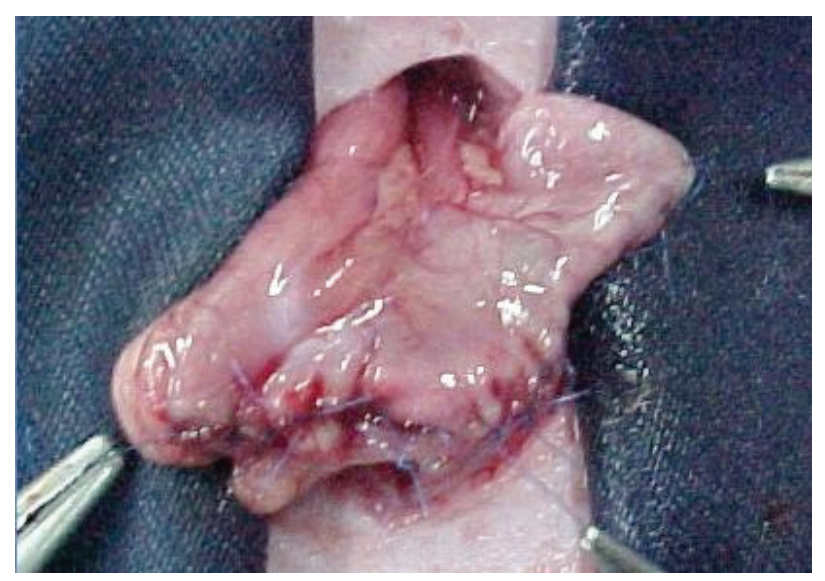

FIGURA 1 - Gastrorrafia

O fornecimento de água iniciou-se imediatamente após a recuperação anestésica e o de dieta a partir de 24 $\mathrm{h}$ de pós-operatório até $12 \mathrm{~h}$ antes da morte. Os animais dos grupos $\mathrm{C} 4$ e $\mathrm{O} 4$ foram mortos no $4^{\circ}$ dia de pósoperatório, enquanto os animais dos grupos $\mathrm{C} 7$ e $\mathrm{O} 7$ no $7^{\circ}$ dia de pós-operatório. No dia previamente determinado, os animais foram mortos com dose letal de éter etílico e submetidos à laparotomia mediana. Posteriormente, procedeu-se a retirada de todo o estômago do animal e os espécimes foram separados para avaliação tensiométrica e histológica.

A avaliação da força de tração e de ruptura da sutura gástrica foi realizada no próprio Laboratório de Cirurgia Experimental de Pós-Graduação em Cirurgia da UFPR, utilizando a máquina universal de ensaio mecânicocomputadorizada, com garras de pressão ajustável manualmente e sistema eletrônico de aquisição de dados com utilização do "software". O segmento gástrico contendo a sutura em sua porção média foi fixado em suas extremidades às presilhas com ajuste manual. A tração ocorreu com velocidade de $50 \mathrm{~mm} /$ minuto, utilizando-se carga de $10 \mathrm{~kg}$, com sensibilidade de $50 \mathrm{~g}$, com margem de erro de $0,5 \%$, até a ruptura total do corpo de prova. A força de tração foi constantemente mensurada através do computador anexo ao sistema de presilhas. As variáveis consideradas no estudo foram: a força máxima de tração (FMT) em $\mathrm{kgf} / \mathrm{cm}^{2}$ e a força de ruptura total (FRT) em kgf (Figura 2). 


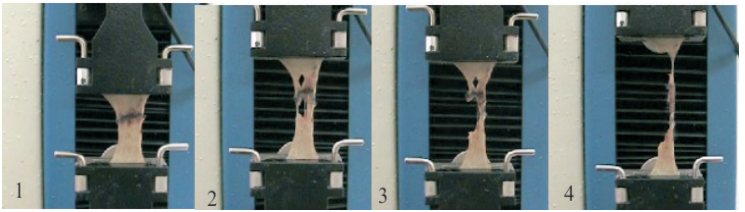

Nota: Seqüência do estudo demonstrando a FMT e FRT nos corpos de prova contendo a sutura gástrica.

FIGURA 2 - Estudo de tração da sutura gástrica

O programa demonstra um sistema de coordenadas cartesianas, cujo eixo x representa a unidade distância e o eixo y a da força (Figura 3). Inicialmente o gráfico apresentou direção ascendente. No momento da ruptura inicial, houve mudança imediata de direção correspondendo à FMT suportada pelo corpo de prova. Durante a fase descendente do gráfico, a ruptura da peça estava em progressão, até o momento de queda de tensão ao nível zero; este ponto correspondeu à separação total dos cotos gástricos. A última força medida pelo computador, antes da separação total dos

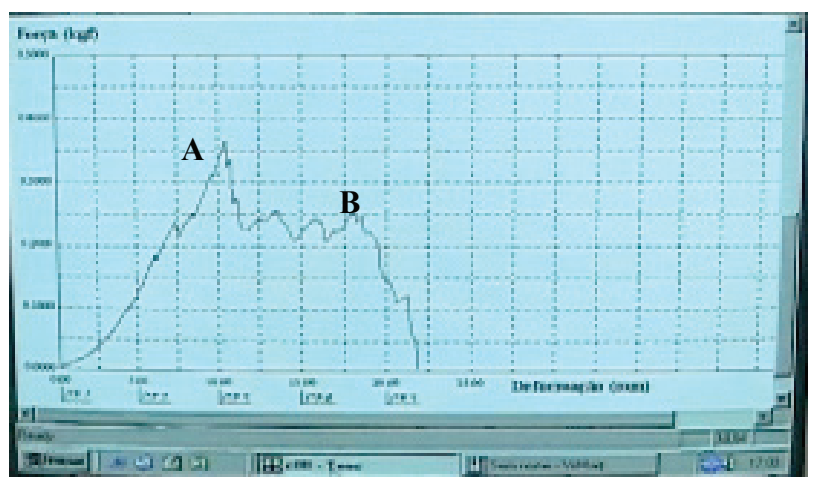

Legenda: $\mathrm{A}=$ ponto correspondente a FMT e B= ponto correspondente a FRT

FIGURA 3 - Gráfico do estudo de tração nos corpos de prova

corpos, no gráfico, foi denominada FRT (Figura 3).

Para a análise qualitativa do colágeno foi obtido fragmento longitudinal de $5 \mathrm{~mm}$ do corpo, compreendendo a sutura gástrica. Estas amostras foram conservadas em frascos separados em formol a $10 \%$. Após microtomia, os cortes histológicos foram submetidos à coloração pelo picrosiriusred - F3BA. Para identificação de colágenos maduro (tipo I) e imaturo (tipo III), analisaram-se os cortes histológicos por meio de microscópio óptico, em aumento de 400 vezes, com fonte de luz polarizada. As imagens foram captadas por uma câmera e transmitidas a monitor colorido, congeladas e digitalizadas por meio de placas. Finalmente, foi realizada a análise das imagens mediante o aplicativo Image Pro-Plus versão 4.5 para Windows (Mídia Cibernética, São Paulo, SP). No sistema RGB (Red, Blue, Green) foram considerados valores para tons de vermelho, amarelo e laranja (colágeno tipo I) e para tons de verde (colágeno tipo III) (Figura 4). Todas as lâminas foram avaliadas sob as mesmas condições de regulagem, dentro dos parâmetros exigidos pelo referido aplicativo. Nos cortes histológicos analisados determinouse o campo microscópico sobre a linha de sutura e foram obtidas medidas de duas áreas distintas. Em cada uma delas o "software" calculou a percentagem da área ocupada pelas fibras que continha colágeno dos tipos I e III em relação à área total examinada. Considerou-se como medida da percentagem de fibras colágenas, a média das duas áreas analisadas.

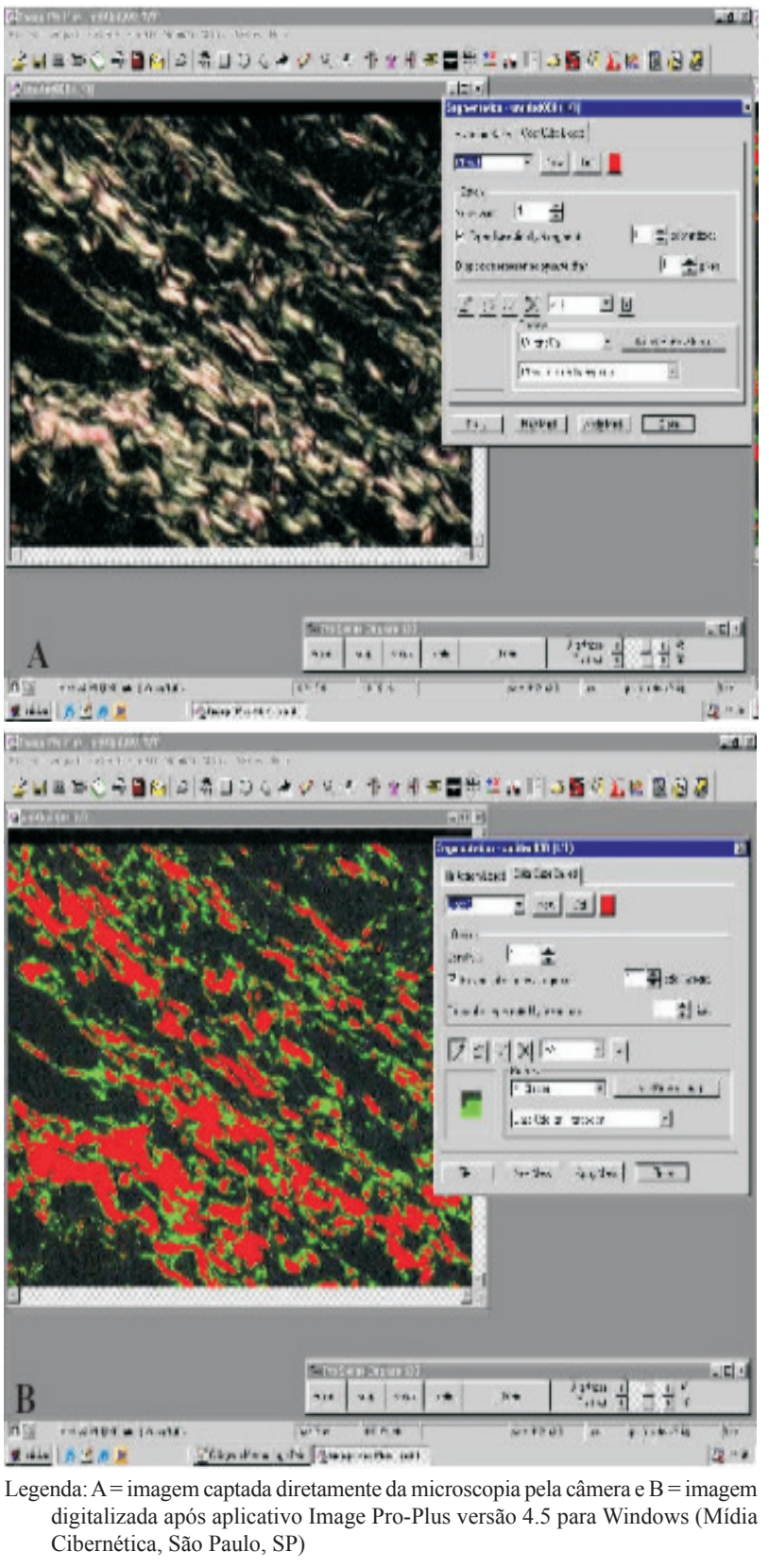

FIGURA 4 - Achados histológicos digitalizados para análise de deposição do colágeno na sutura gástrica

A partir da medida da percentagem de fibras colágenas foi calculado o índice de maturação de colágeno (IMaC) (Quadro 1), onde valores maiores do que 1 indicam que a percentagem de colágeno tipo I é maior do que a percentagem de colágeno do tipo III.

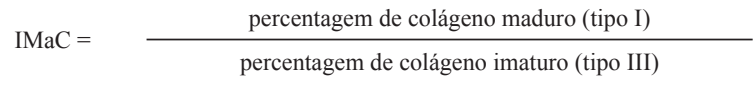

Adaptado de Coelho-Lemos et al. 2003.

QUADRO 1 - Fórmula para o cálculo do índice de maturação do colágeno - IMaC 
Procedeu-se o tratamento estatístico, de acordo com a natureza dos dados estudados. Os resultados foram expressos em média \pm erro padrão $(\mathrm{M} \pm \mathrm{EP})$. $\mathrm{Na}$ análise não-paramétrica foi utilizado o teste de Kruskal-Wallis e na análise paramétrica o teste $t$ de Student. Em todos os testes, o nível de significância adotado foi $P<0,05$.

Os animais em que foram encontrados sinais de abscesso, fístula, peritonite, bem como os que evoluíram a óbito durante o experimento foram excluídos de todas as avaliações subseqüentes do estudo.

\section{RESULTADOS}

Dos 44 animais do estudo, 5 foram excluídos da análise estatística, 3 por terem evoluído a óbito no $1^{\circ}$ e $3^{\circ}$ dias do pós-operatório, respectivamente, por causa não determinada na necropsia e outros 2 animais por apresentarem alto índice de aderências. Trinta e nove animais do estudo foram incluídos na análise estatística, subdivididos em: GC4, GC7, GO4 e GO7.

O peso inicial médio dos animais avaliados foi de $255,95 \pm 3,03 \mathrm{~g}$. Não foi evidenciada diferença estatisticamente significante entre os grupos. Todos os animais apresentaram perda ponderal após o procedimento cirúrgico, porém sem significado estatístico (Gráfico 1).

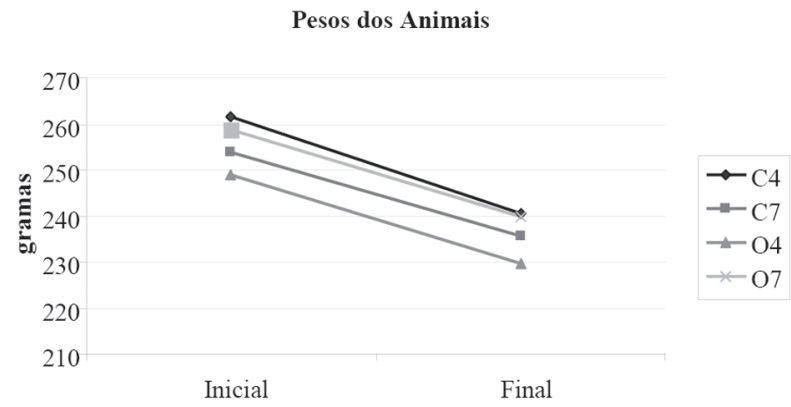

Nota: pesos expressos em gramas. $* P>0,05$

Legenda: $\mathrm{C}=$ grupo controle e $\mathrm{O}=$ grupo octreotide $/ 4=4^{\circ}$ dia do pós-operatório e $7=7^{\circ}$ dia do pós-operatório

GRÁFICO 1- Pesos dos animais

Todos os corpos de prova considerados no presente estudo apresentaram padrões de ruptura na linha de anastomose. A força máxima de tração (FMT) foi analisada por comparação das médias. Houve diferença estatística somente no grupo controle, quando comparado a média das FMT entre o $4^{\circ}$ e $7^{\circ}$ dias, com valores de FMT maiores no $7^{\circ}$ dia (Tabelas 1 e 2 ).

TABELA 1 - Comparação das médias da FMT intergrupos e intragrupos

\begin{tabular}{ccccc}
\hline Grupo & FMT $(\mathrm{M} \pm \mathrm{EP})$ & Grupo & FMT $(\mathrm{M} \pm \mathrm{EP})$ & $P$ \\
\hline C4 & $1,333 \pm 0,101$ & O4 & $1,667 \pm 0,155$ & $P^{*}=0,0800$ \\
$\mathrm{C} 7$ & $1,985 \pm 0,292$ & O7 & $1,777 \pm 0,144$ & $P^{*}=0,4500$ \\
\multicolumn{2}{c}{$P^{* *}=0,4500$} & & $P^{* *}=0,8200$ & \\
\hline
\end{tabular}

Legenda: M- média, EP- erro-padrão, $P$ *- nível de significância estatística entre $\mathrm{C} 4$ e $\mathrm{O} 4$ e entre $\mathrm{C} 7$ e O7, $P^{* *}$ - nível de significância estatística entre $\mathrm{C} 4 \mathrm{e}$ $\mathrm{C} 7$ e entre $\mathrm{O} 4 \mathrm{e} \mathrm{O} 7$
TABELA 2 - Comparação das médias da FRT intergrupos e intragrupos

\begin{tabular}{ccccc}
\hline Grupo & FRT $(\mathrm{M} \pm \mathrm{EP})$ & Grupo & FRT $(\mathrm{M} \pm \mathrm{EP})$ & $P$ \\
\hline C4 & $0,213 \pm 0,020$ & O4 & $0,184 \pm 0,027$ & $P^{*}=0,4000$ \\
C7 & $0,214 \pm 0,027$ & O7 & $0,174 \pm 0,034$ & $P^{*}=0,4700$ \\
\multicolumn{2}{c}{$P^{* *}=0,4500$} & & $P^{* *}=0,8200$ & \\
\hline
\end{tabular}

Legenda: M- média, EP- erro-padrão, $P$ *- nível de significância estatística entre $\mathrm{C} 4$ e $\mathrm{O} 4$ e entre $\mathrm{C} 7$ e O7, $P * *$ - nível de significância estatística entre $\mathrm{C} 4 \mathrm{e}$ $\mathrm{C} 7$ e entre $\mathrm{O} 4 \mathrm{e} \mathrm{O} 7$

Na avaliação da densitometria do colágeno, não houve diferença estatisticamente significativa comparando-se as médias das áreas percentuais das suturas gástricas em todos os grupos com relação ao colágeno imaturo (tipo III). Analisando estatisticamente as médias de percentual de colágeno maduro (tipo I) houve, dentro dos grupos, maior percentual deste tipo de colágeno no grupo controle de $7^{\circ}$ dia do pós-operatório (média $21,43 \pm 1,06$ ) em relação ao controle de $4^{\circ}$ dia do pós-operatório (média 11,26 $\pm 1,44$ ) com $P=0,0000$. Comparando-se os valores de $4^{\circ}$ e $7^{\circ}$ dia do pós-operatório no grupo octreotide, não houve diferença estatística. Quando comparados o percentual de colágeno maduro entre os grupos, notou-se diferença estatística no $4^{\circ}$ dia com percentagem maior no grupo que recebeu octreotide (média 19,13 $\pm 1,38$ ) em relação ao controle (média $11,26 \pm 1,44) \operatorname{com} P=0,001$.

A partir dos valores de percentuais de colágeno imaturo e maduro das amostras de sutura gástrica, procedeu-se ao cálculo do IMaC. Nos dados analisados foram encontradas médias de $\mathrm{IMaC}$ acima de 1,0 nos grupos controle $7^{\circ}$ dia e octreotide no $4^{\circ}$ e $7^{\circ}$ dias (Gráfico 2).

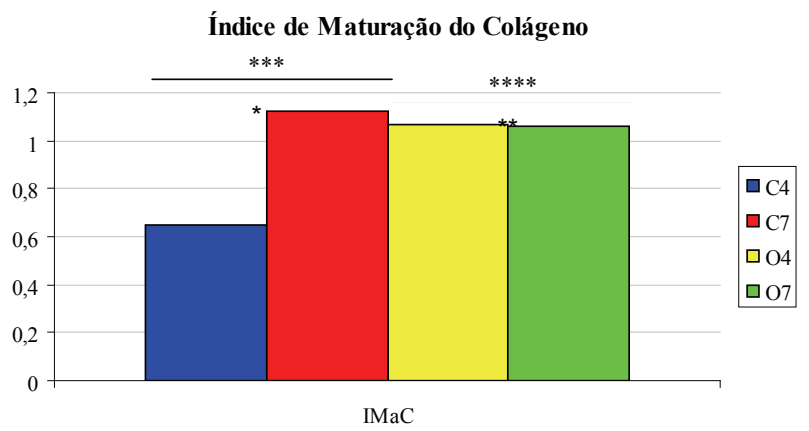

Legenda: $\mathrm{C} 4=$ grupo controle $4^{\circ}$ dia do pós-operatório; $\mathrm{C} 7=$ grupo controle $7^{\circ}$ dia do pós-operatório; $\mathrm{O} 4=$ grupo octreotide $4^{\circ}$ dia do pós-operatório; $\mathrm{O} 7=$ grupo octreotide $7^{\circ}$ dia do pós-operatório * $P=0,0001$; ** $P>0,5 ; * * * P=0,001$; $* * * * P>0,5$

GRÁFICO 2 - Índice de maturação do colágeno

\section{DISCUSSÃO}

Poucos estudos experimentais avaliando a cicatrização de anastomoses gástricas e a aplicação de octreotide foram identificados na literatura ${ }^{1,6,16}$. Entretanto, não foram vistos trabalhos avaliando a influência do octreotide na cicatrização de suturas gástricas.

O octreotide é potente inibidor de inúmeros hormônios (hormônio do crescimento, TSH, insulina, glucagon e hormônios intestinais), de secreções exócrinas (ácido gástrico, enzimas pancreáticas) e de absorção do intestino delgado, 
podendo ser empregado na prática clínica para uma série de desordens ${ }^{19,25}$. A administração de octreotide mostrou ser efetiva tanto na via endovenosa (bolus ou infusão contínua) como na via subcutânea (dose única ou fracionada) em vários estudos experimentais em ratos ${ }^{13,15,16}$.

A via de administração escolhida foi a subcutânea tendo em vista a praticidade de aplicação no pré e pós-operatório. As dosagens desta medicação também variam em estudos experimentais. Neste experimento, a dosagem de octreotide escolhida foi de $20 \mu \mathrm{g} / \mathrm{kg} /$ dia, em aplicação única diária subcutânea, sendo a primeira dose antes do procedimento cirúrgico $^{24,25}$. Doses inferiores a $20 \mu \mathrm{g} / \mathrm{kg} /$ dia por essa via mostraram-se ineficazes e doses superiores causaram efeitos adversos ${ }^{16,23,24}$.

Os parâmetros mecânicos são utilizados com freqüência em estudos de cicatrização de suturas. A resistência da anastomose às tensões normais do organismo pode ser avaliada pela força de ruptura (tração das extremidades) ou pela força ou pressão de explosão (resistência da ferida cirúrgica à insuflação gasosa ou de líquidos). As forças de explosão e de ruptura representam forças que atuam em diferentes direções nas suturas. Estudos anteriores utilizaram o equipamento para estudo de tração, com garras de pressão pneumática ${ }^{4,22,26}$. No presente estudo foi utilizado equipamento semelhante, porém com garras de ajuste manual, a fim de não lacerar o corpo de prova durante o estudo da tração ${ }^{17}$. Porém, ainda existe controvérsia entre os autores sobre qual método seria o mais fidedigno para se avaliar a resistência de anastomose. A força de explosão representa a força exercida pela pressão intraluminal que, por sua vez, representa a força natural que atua sobre as suturas intestinais ${ }^{13}$. No presente estudo foram escolhidos como parâmetros mecânicos da avaliação da sutura gástrica a medida de força máxima de tração e a medida da força de ruptura total, baseando-se nos estudos encontrados na literatura ${ }^{6,7,17}$ e em experimentos realizados previamente no Programa de Pós-Graduação em Clínica Cirúrgica ${ }^{1,3,4,5,22,26}$, nos quais foi observado que, após o $7^{\circ}$ dia de pós-operatório, a medida de força de ruptura foi semelhante ao grupo controle.

Diversos estudos avaliaram a influência do octreotide na resistência das anastomoses intestinais. Miller et al. ${ }^{15}$ estudaram os efeitos do octreotide na cicatrização das anastomoses de intestino delgado no $7^{\circ}$ dia do pósoperatório e concluíram que a administração de octreotide não influenciou a força de explosão, mesmo em doses altas. Já Turkçapar et al. ${ }^{25}$ compararam os valores de pressão de explosão na cicatrização de anastomoses colônicas em ratos no $5^{\circ}$ e $8^{\circ}$ dias do pós-operatório nos grupos controle, octreotide e esteróide. Este estudo concluiu que a pressão de explosão no grupo octreotide no $5^{\circ}$ dia do pós-operatório foi significativamente menor que nos outros grupos $(P=0.001)$, e que no $8^{\circ}$ dia ela nos grupos esteróide e octreotide foi significativamente menor do que o grupo controle $(P=0.001)^{25}$. Seguindo o mesmo modelo, Papalambros et al. ${ }^{18}$ estudaram a influência do uso de octreotide subcutâneo após ressecção de intestino delgado. Os resultados deste estudo mostraram aumento na pressão de explosão das anastomoses no grupo octreotide no $8^{\circ} \mathrm{e}$ $15^{\circ}$ dias do pós-operatório, com $P<0,05^{18}$. No presente estudo, não se observou diferença estatística entre os grupos controle e octreotide na análise das médias de FMT, no $4^{\circ}$ e $7^{\circ}$ dia do pós-operatório. Houve diferença nas médias de FMT no grupo C4 (média 1,333 $\pm 0,01$ ) em comparação a C7 (média $1,985 \pm 0,292$ ) com $P=0,03$, demonstrando aumento da resistência esperada com a evolução pós-operatória. A administração de octreotide não mostrou prejuízo à FMT. Analisando-se os valores de força de ruptura intergrupos e intragrupos, não houve diferença estatística entre eles.

Estudos comparando diversos métodos de coloração para análise do colágeno, concluíram que o corante picrosirius-red é de simples execução, fácil interpretação e específico para o estudo do colágeno tecidual, uma vez que torna suas fibras birrefringentes à luz polarizada, possibilitando, assim, a sua quantificação na amostra analisada. As fibras de maior espessura (colágeno do tipo I) aparecem fortemente birrefringentes e fibras de menor espessura (colágeno do tipo III) fracamente birrefringentes. Esta diferenciação de cores permite análise qualitativa das fibras colágenas, obtendo-se a densitometria de fibras maduras (colágeno tipo I) e imaturas (colágeno tipo III) que, em última análise, refletem a proporção de entrelaçamento das fibras ${ }^{10,11}$. A análise histopatológica no presente estudo foi realizada com o método de coloração de PCS, baseado nos estudos prévios que ressaltam a importância de se obter análise qualitativa do colágeno ${ }^{1,3,4,5,20}$.

Gottrup, em $1981^{7}$, verificou a distribuição de colágeno por análise quantitativa, correlacionando-a à força de ruptura mecânica em sutura gástrica em ratos e concluiu que a concentração de colágeno é fator primordial na propriedade mecânica da cicatrização de sutura gástrica em ratos. Da mesma forma, Bonin et al. ${ }^{1}$ analisaram o depósito de colágeno em anastomoses gástricas em ratos que receberam pantoprazol. No grupo que o observou-se maior proporção de colágeno tipo I em relação ao tipo III no corpo (epitélio gástrico glandular) após 4 e 7 dias de pós-operatório e no rumen (epitélio gástrico não-glandular) após 7 dias de pós-operatório em ratos submetidos à sutura gástrica ${ }^{1}$. No presente estudo observou-se que a percentagem de depósito de colágeno do tipo III (imaturo) foi maior no grupo C4, porém sem diferença estatística entre os grupos e dentro dos grupos. Quando os grupos de $4^{\circ}$ dia do pós-operatório foram comparados, notou-se maior concentração de colágeno maduro no grupo que recebeu octreotide em relação ao controle, traduzindo maior organização e espessamento das fibras colágenas.

Neste experimento, a despeito da tendência à maior deposição de colágeno no $4^{\circ}$ dia de pós-operatório com o uso do octreotide, este resultado não se repetiu na análise das médias da FMT e da força de ruptura. A correlação positiva entre quantificação de colágeno e força de ruptura é motivo de controvérsia. Coelho-Lemos et al. ${ }^{3}$ realizaram estudo, em 2004, para verificar a influência da desnutrição intra-uterina na cicatrização da parede abdominal em ratos lactentes, e idealizaram o índice de maturação do colágeno (IMaC). No presente estudo, observou-se valores positivos (acima de 1) nos grupos C7, O4 e O7. Estatisticamente notou-se valores de IMaC maiores no grupo que recebeu 
octreotide no $4^{\circ}$ dia do pós-operatório. No grupo controle, observou-se valores positivos de IMaC no grupo $\mathrm{C} 7$; no grupo octreotide não se notou diferença estatística entre $\mathrm{o}$ grupo $\mathrm{O} 4$ e $\mathrm{O} 7$, porém nestes dois grupos encontraram-se valores positivos de IMaC.

Todos estes achados devem ser corroborados com mais estudos, pois existem controvérsias no que diz respeito à correlação positiva entre a maior quantidade de colágeno e a maior resistência do tecido à força de ruptura, principalmente quando se avalia a $1^{\circ}$ semana do pós-operatório ${ }^{3,7}$. Embora existam muitos estudos sobre a influência, deletéria ou não, do octreotide sobre anastomoses colônicas e intestinais, não foram identificados trabalhos na literatura a respeito dos efeitos da administração de octreotide sobre o metabolismo do colágeno e na resistência à força de tração e ruptura de suturas gástricas.
Outros experimentos evidenciaram a redução do fluxo portal e esplâncnico, o que poderia influenciar negativamente a cicatrização ${ }^{2}$; porém, no presente estudo, não foi evidenciado tal fato, pelo menos na primeira semana do pós-operatório. Os resultados aqui apresentados sugerem que o octreotide poderia ser utilizado com segurança no tratamento de fístulas digestivas ou de sangramento digestivo em pacientes com anastomoses recentes, pois o seu uso não se associou à redução da força tênsil da sutura gástrica.

\section{CONCLUSÕES}

A administração subcutânea de octreotide em ratos submetidos à sutura gástrica aumentou a percentagem de deposição de colágeno maduro no $4^{\circ}$ dia do pós-operatório, porém não alterou a resistência da sutura à tensão.

Trubian PS, Branco AB, Campos ACL, Ferreira M, Trubian PS, Matias JEF.Effect of octreotide administered subcutaneously on the healing of gastric sutures in rats. ABCD Arq Bras Cir Dig 2007;20(3):172-8.

ABSTRACT - Background - Octreotide, a somatostatin synthetic analogue, is a potent inhibitor of gastric intestinal and pancreatic secretions. It also inhibits many trophic and anabolics hormones, decreases splanchnic blood flow, accelerates gastric emptying and prolongs intestinal transit time, increasing the absorption of water and electrolytes. Aim - Evaluate the effects of octreotide on gastric sutures in rats. Methods - Thirty-nine male adult Wistar rats were used, divided into control group and octreotide group. They were further subdivided into two subgroups, according to the day they were killed, the 4th or the 7th postoperative day. All animals were submitted to gastric longitudinal incisions, were closed using seromuscular interrupted stitches. The animals from the octreotide group received subcutaneous injections of octreotide. Control group received $0,9 \%$ isotonic saline. The samples of the stomach were obtained in order to determine the percentage of mature collagen and immature collagen, by picrosirius-red F3BA technique, the collagen maturation index and to determine the breaking strength and maximum strength force, tested in a computerized tensiometer. For statistical analysis, Student's $t$ and Kruskall-Wallis' tests were applied, with the level of significance set at $P<0.05$. Results - There was no difference in the percentage of immature collagen beeween the groups. The deposition of mature colagen was higher in the control group on the 7 th postoperative day $(21,43 \pm 1,06$ vs.11,26 $\pm 1,44, P=0,0000)$; in the octreotide group there was no difference. Comparing control and octreotide groups the deposition of mature collagen is higher in the octreotide group on the 4th postoperative day $(19,13 \pm 1,38$ vs. $11,26 \pm 1,44, P=0,001)$. The collagen maturation index was higher in the octreotide group on the 4 th postoperative day $(1,07 \pm 0,07$ vs $0,65 \pm 0,05$, $P=0,0001$ ). The maximum strength force and the breaking strength were similar between control and octreotide groups. Conclusions -Octreotide given subcutaneously in rats is associated with higher proportion of mature collagen and does not influence the maximum strength force and the breaking strength of gastric sutures in rats.

HEADINGS - Octreotide. Collagen.

\section{REFERÊNCIAS}

1. Bonin EA, Campos ACL, Coelho JCU, Matias JEF, Malafaia O, Jonasson TH. Effect of pantoprazole administered subcutaneously on the healing of sutured gastric incisions in rats. Eur Surg Res. 2005;37:250-6.

2. Campos ACL, Costa MAR, Bonin EA, Druszcz CC, Czeczko NG, Coelho JCU. Uso da octreotida como adjuvante no tratamento das fístulas digestivas. Rev Med Paraná. 2001;59:43-7.

3. Coelho-Lemos ICM, Campos ACL, Almeida M, Malafaia O, Schüler SL, Gurmini J. In-utero malnutrition influences wound healing of newborn rats as measured by tensile strength and collagen deposition. JPEN J Parent Enteral Nutr. 2004;28:241-4.

4. Costa MAR, Campos ACL, Coelho JCU, Barros AM, Matsumoto HM. Oral glutamine and the healing of colonic anastomoses in rats. JPEN J Parent Enteral Nutr. 2003;27:182-5.

5. Gonçalves CG, Campos AC, Groth AK, Ferreira M, Coelho, JCU, Meguid, MM. Influence of preoperative feeding on the healing of colonic anastomosis in malnourished rats. JPEN J Parent Enteral Nutr. 2005;29(1 Suppl):1-48.

6. Gottrup F. Healing of incisional wounds in stomach and duodenum: a biomechanical study. Am J Surg. 1980;140:296-301.

7. Gottrup F. Healing of incisional wounds in stomach and duodenum - collagen distribution and relation to mechanical strength. Am J Surg. 1981;141:2227.

8. Harris A. Somatostatin and somatostatin analogues: pharmacokinetics and pharmacodynamic effects. Gut. 1994;35:1-4.

9. Jiborn H, Ahonen J, Zederfeldt B. Healing of experimental colonic anastomosis. Effect of suture technique on collagen concentration in the colonic wall. Am J Surg. 1978;135:333-40.
10.Junqueira LC, Bignolas G, Brentani RR. Picrosirius staining plus polarization microscopy, a specific method for collagen detection in tissue sections. Histochemistry J.1979;11:447-55.

11. Junqueira LC, Montes GS, Sanchez EM. The influence of tissue thickness on the study of collagen by the picrosirius-polarization method. Histochemistry. 1982;74 (Suppl 1):153-6.

12. Koruda MJ, Rolandelli RH. Experimental studies on the healing of colonic anastomosis. J Surg Res. 1990;48:504-15.

13. Kubba AK, Dallal H, Haydon GH. The effects of octreotide on gastroduodenal blood flow measured by laser Doppler flowmetry in rabbits and man. Am J Gastroenterol. 1999;94:1077-82.

14. Medeiros AC, Mello NM, Macedo LM, Medeiros IC, Dantas Filho AM, Aires Neto T. Octreotida no tratamento de fístulas entéricas de ratos. Acta Cir Bras. 2002; 17:116-21.

15. Miller SK, Martindale RG, Gao XX, Gadacz TR. The effects of octreotide on healing of small bowel anastomosis. Am Surg. 1996;9:733-7.

16. Motegi M, Nagamachi Y, Kaneko T. Acute in vivo effect of octreotide acetate, a somatostatin analogue on the cellular function of gastric mucosa in the rat. J Gastroenterol Hepatol. 1998;13:220-4.

17. Naresse LE, Mendes EF, Curi PR, Lucchiari PH, Kobayashi S. Aparelho para medida de força de ruptura das anastomoses intestinais. Rev Hosp Clin Fac Med S Paulo. 1987;42:204-8.

18. Papamambros E, Filis K, Angelopoulou R. Does octreotide impair anastomotic healing after small bowel resection? Acta Chir Belg. 2004;104:198-203.

19. Prevelik GM. Clinical use of octreotide (Sandostatin) in endocrinology. Med Pregl. 1993;46:343-8. 
20. Rabau MY, Dayan D. Polarization microscopy of picrosirius-red stained sections: a useful method for qualitative of intestinal wall collagen. Histol Histopath. 1994;9:525-8.

21. Savassi-Rocha PR, Lopes RL. Anastomoses intestinais: bases da cicatrização e análise dos diferentes tipos. In: Castro LP, Savassi-Rocha PR, Cunha-Mello JR. Tópicos em Gastroenterologia. Rio de Janeiro: 1994;5:493-521.

22. Skinovskyn J. A influência da nicotina na cicatrização de anastomoses do intestino delgado em ratos. [Dissertação]. Curitiba: Setor Ciências da Saúde - Universidade Federal do Paraná; 2000.

23. Thompson JS, Nguyen BT, Harty RF. Somatostatin analogue inhibits intestinal regeneration. Arch Surg. 1993;128:385-9.
24. Tocchi A, Costa G, Lepre L, Liotta G, Mazzoni E, Miccini M. Effects of octreotide (somatostatina analog SMS 201-995) on superior mesenteric artery bood flow in swine. An experimental study using Doppler color ultrasonography. G Chir. 1999;20:9-13.

25. Turkçapar AG, Demirer S, Sengul N, Ersoz S, Kuterderm E, Renda N, Kuzu I. The adverse effects of octreotide on the healing of colonic anastomoses in rats. Surg Today. 1998;28:279-84.

26. Vizzoto Junior AO, Campos AC, Vieira JF, Silva ES, Malafaia O, Czeczko $\mathrm{NG}$, Noronha L. Influence of cisplatin given pre and postoperatively on the tensile strength of colonic anastomosis in rats. ABCD - Arq Bras Cir Dig. $2002 ; 15 ; 49-53$

Conflito de interesse: não há Fonte financiadora: não há Recebido para publicação em: 03/05/2007 Aceito para publicação em: 16/06/2007 